\title{
Research on Risk Diffusion Mechanism of Logistics Service Supply Chain in Urgent Scenarios
}

\author{
Guangsheng ZHANG $\mathbb{D}^{1,2}$ Xiao WANG ${ }^{10},{ }^{3}$ Zhijun GAO, ${ }^{4}$ and Tianyu XIANG ${ }^{3}$ \\ ${ }^{1}$ School of Management, Zhejiang University of Technology, Hangzhou 310014, China \\ ${ }^{2}$ College of Business Administration, Shandong Management University, Jinan 250357, China \\ ${ }^{3}$ College of International Business, Xi'an Jiaotong-Liverpool University, Suzhou 215123, China \\ ${ }^{4}$ School of Transport \& Communication, Shanghai Maritime University, Shanghai 201306, China \\ Correspondence should be addressed to Xiao WANG; xiao.wang@xjtlu.edu.cn
}

Received 21 August 2019; Revised 9 December 2019; Accepted 27 January 2020; Published 28 February 2020

Academic Editor: Mariano Torrisi

Copyright ( $\odot 2020$ Guangsheng ZHANG et al. This is an open access article distributed under the Creative Commons Attribution License, which permits unrestricted use, distribution, and reproduction in any medium, provided the original work is properly cited.

\begin{abstract}
Although there are some studies on the risk management of logistics service supply chain (LSSC), we still know little about the effects of risk evolution and interactional mechanism of subjects in different urgent scenarios of logistics service supply chain from a micro perspective. Based on the structures of the LSSC, this study thus analyzed the risk evolution and the main actors' reactions in the LSSC, including categorizing the urgent scenarios, analyzing their impacts on the diffusion intensity of pricing, profit and demand, and verifying the models' validity with the numerical analysis. The results indicate divergent impacts of the different urgent scenarios on the logistics service supply chain: the price fluctuation can cause similar profit declines of the supplier and integrator, the reduced capacity can cause the supply chain's price increase by $50 \%$, and the demand risks can lead to similar changes of the upstream orders, prices and profits. The findings may provide clues for firms to develop effective countermeasures against the risks.
\end{abstract}

\section{Introduction}

To cope with increasingly fierce competition, supply chain firms in Japan, Europe, and the U.S. have taken the lead in switching to Just-in-Time production and lean management, adopting the model of centralized production and distribution. Companies have outsourced their internal logistics business to professional and highly efficient logistics service providers, with lower cost and higher efficiency, thus focusing more on gaining and maintaining its core competitiveness. Different from a product supply chain, logistics services have to be delivered to customers in real time according to their final needs, and logistics outsourcers thus have to be able to adjust their service strategies in time according to the production pace of their customers (enterprises). Logistics Service Supply Chain (LSSC), a new management model with the basic structure of "functional logistics service provider $\longrightarrow$ logistics service provider $\longrightarrow$ logistics users," facilitates the efficient operation of product supply chain by coordinating the supply and demand mechanism of logistics services [1]. However, with intensifying market competition and the trend of globalization and personalized development, logistics services can be easily affected by internal and external factors, and the urgent scenarios may inevitably interfere the implementation of service plan, causing failure in the interenterprise services delivery [2]. Although the lean, just-in-time system can effectively improve operational efficiency, reduce cost, and encourage closer cooperation among enterprises, it has also increased the vulnerability of a supply chain system: a single enterprise's urgency tend to affect any related nodes, bringing great losses to the supply chain system by impeding promised logistics service provision and thus causing great losses to all of the related enterprises. In particular, in the highly volatile internal and external business environment of China, with the urgencies enterprises are prone to face 
greater challenges of risk management. Studying the risk diffusion mechanism of the logistics service supply chain in the urgent scenarios thus is of critical significance.

In recent years, with the intensifying globalization, deteriorating economic environment, and popularizing lean management in enterprises, the supply chain structure of enterprises is quite fragile in various urgencies. Gedik et al. [3] and Paloviita et al. [4] pointed out that the increasingly lean and agile trend of supply chain aggravates the vulnerability of the supply chain network. Firstly, on the basis of the risk transmission mechanism of the supply chain in urgencies, many researchers have investigated the transmission and diffusion effect of the demand fluctuation and supply interruption in a supply chain. Christopher [5] studied the logistics service elements of the supply chain, pointed out that logistics is an important strategy to realize the supply chain value, and analyzed the diffusion intensity of supply and demand risks from the perspectives of cost, service and customer value. Ivanov [6] covered recent developments in supply chain risk management and resilience from the position of structural dynamics focusing on the disruption propagation and the ripple effect. Qazi et al. [7] developed a supply chain risk network management process that captured interdependencies between risks, multiple performance measures, and risk mitigation strategies within a network setting. Ojha et al. [8] attempted to measure risktaking following the assessment of supply chain risk propagation, and bayesian network theory is used to analyze the multiechelon network faced with simultaneous disruptions. Liang'an et al. [9] proposed a new model for supply chain risk propagation considering herd mentality and risk preference under warning information on multiplex networks, in which one layer is used to denote the risk propagation and the other one represents the warning information propagation network. The above research results systematically analyzed the generation and diffusion of supply chain urgencies, which helped us to understand diffusion intensity and evolution effect of urgency risks in the logistics service supply chain from different perspectives. Nevertheless, there is still lack of insights pertaining to supply chain enterprises' behaviors on a micro level, especially what influences different variables (e.g., different transmission time and scope) would have on the major players of a supply chain.

Although scholars have developed many strategies for handling the issues caused by the urgencies, they mainly focus on implementing multisource procurement, strengthening supplier tracking, optimizing cooperative relations, establishing spare production lines, and improving the supply chain transparency. In the early stage, Berger et al. [10] studied the single supplier disruption and all supplier disruptions, and adopted the decision tree model to determine the optimal number of suppliers. Kumar et al. [11] studied how a retailer can use pricing decisions along with sourcing strategies under disruption risk while competing against another retailer with a more reliable supply chain. Bimpikis et al. [12] provided an explicit characterization of equilibrium profits, which allows us to derive insights into how the network structure, that is, the number of firms in each tier, production costs, and disruption risk-affect firms' profits. Wang and Yin [13] considered three common backup strategies between the firm and the backup supplier: advance purchase, reservation, and contingency purchase. In the traditional supply chain risk research, using mathematical optimization to solve the problem of supply risk has a good research foundation, but it does not involve relevant research on the risks of logistics services supply chain, which is more aligned to our research theme.

Since the supply chain is an integrated system composed of various node-based member enterprises, some scholars have studied supply chain issues based on the urgencies' impact on the internal operation risk of the supply chain. Fattahi et al. [14] proposed the scheme of an elastic network strategy for the internal operation risk in the multiphase supply chain network design. Kwak et al. [15] proposed and validated a theoretical model to investigate whether supply chain (SC) innovation positively affects risk management capabilities, such as robustness and resilience in global SC operations, and to examine how these capabilities may improve competitive advantage. Ivanov et al. [16] studied the impact of digitalisation and Industry 4.0 on the ripple effect and disruption risk control analytics in the supply chain. However, studies based on the urgency risk of supply chain operation are relatively insufficient, where structuring a system of the LSSC comparatively still lacks investigation. Largely, the supply chain operation mechanism can reveal the developmental interactions between microscopic behaviors and macroscopic phenomena and can depict the main heterogeneity and different dynamic behaviors to reveal the essence behind the evolution of risks.

Furthermore, the urgency risk caused by sudden demand has always been a hot and difficult issue in the supply chain field. Qi et al. [17] carried out research early and suggested "supply chain coordination with demand disruptions." Following this research, Huang and Wang [18] investigated disruptions of market demand and supply quantity of used products in a closed-loop supply chain where the manufacturer licenses the third party to undertake remanufacturing activities. Yan et al. [19] showed that the amount of change in decision-making is a linear function of the amount of demand disruption, and it is independent of the riskaverse coefficient. Rahmani and Yavari [20] investigated the demand disruption management in a dual-channel supply chain producing and selling green products and examined the pricing and green issues for a dual-channel green supply chain when the market demand is disrupted. They are based on the simplified contract mechanism to coordinate supply chain optimization, where the existing gap between simplified conditions and the reality may affect the feasibility of solutions. There is little literature that adopts multiagent modeling and contract mechanism to study these problems of logistics service supply chain. However, adopting option contract to effectively analyze urgency risk scenarios of the logistics service supply chain and extracting key risk factor indexes to build a multiagent strategy model can provide effective measures for risk management and urgency response plan. 
The overall system of the logistics service supply chain should be adaptable, well-organized, and dynamic. The efficient operation of complex system is of great significance for improving efficiency of the logistics service supply chain network. The above research has deeply discussed diffusion effects of supply risk, operation risk, and demand risk in the product supply chain and has laid a foundation for our research on risk diffusion mechanism of the logistics service supply chain. However, the logistics service supply chain is mainly based on coordination of logistics service capability, which is substantially different from the product supply chain. Strategic heterogeneity directly affects formation and development of cooperative alliances under the risk mechanism. This problem also directly affects the operation efficiency of the logistics service supply chain. With increasing dynamics and complexity of the operational environment, the capability to cope with sudden risk of logistics service can facilitate targeted adjustments against potential risks that undermine corporate profitability, which in turn ensures sustainable operation and customer service. So the aim of this study is to analyze the effects of risk evolution and interactional mechanism of subjects in different urgent scenarios of logistics service supply chain from a micro perspective, and explore the risk diffusion of the logistics service supply chain in urgencies, as well as risk management strategies for supply, operation, and demand urgencies, which are important tasks with practical value and theoretical significance.

In the decision-making process of Stackelberg's masterslave game, higher-level decision-makers exercise control and guidance over lower-level decision-makers, and lowerlevel followers can also deploy decision-making power within their own scope under the guidance. Each level in the master-slave game system has its own objective function, so the final decision result is to enable each decision subject to reach a mutually coordinating scheme. In the logistics service process, the interaction mechanism between functional logistics service providers and logistics service integrators is revealed based on the theory of master-slave game, in which functional logistics service providers are leaders and logistics service integrators are followers. Stackelberg Game theory provides a basic framework for studying diffusion effects of emergency risk. This study mainly focuses on the types of urgencies in the LSSC and constructs the diffusion effect model of the influence of various risk scenarios on the LSSC. Specifically, this study first divided urgent scenarios into supply change, operational failure, and demand change according to the logic of the supply and demand structure of the LSSC. This study analyses the effect of risk evolution and the mechanism of the main actors in the LSSC from a microlevel. Then, the pricing, profit, and demand diffusion intensity at the microlevel of each spot after the occurrence of the urgent risk are investigated. Meanwhile, numerical analysis is used to confirm the effectiveness of the established model. Finally, based on the analytical results, countermeasures are proposed for the prevention and control of urgent risks in the LSSC. Therefore, this research has proved the significance for LSSC enterprises to take effective measures to cope with urgent risks.

\section{Urgency Risk Scenario of Logistics Service Supply Chain}

The implementation of logistics services is often interrupted by various urgencies. These uncertainties will have negative impacts on function supply, service delivery efficiency and customer demand, which will increase the failure risks of the logistics service supply chain system. Based on [21] research on logistics service supply chain urgencies, this study categorizes them into supply risk, operation risk, and demand risk, as shown in Figure 1.

The logistics service supply chain is a complex collaborative network that provides logistics service to ensure logistics operation of the product supply chain. The network nodes of logistics service supply chain represent functional logistics service providers of different functions or different regions, such as providers of warehouse, transportation, and other functions. Function providers offer professional logistics service to integrators through their own services. There are several complete business routes within the logistics service supply chain network where functional logistics service providers complete meeting the logistics service needs through logistics integrators, and there is a clear upstream and downstream sequence among enterprises in the same route. Therefore, this shows that the emergency risk of logistics service supply chain will be affected by several variables such as supply, operation, and demand capacity. Taking the logistics service supply chain structure in Figure 1 as an example, the structure of logistics service capability supply is "logistics service subcontractor-functional logistics service provider," and the functional logistics service provider subcontracts to the upstream logistics service subcontractor according to the service demand of the logistics service integrator. The supply risk is caused by insufficient capacity or failure of the functional suppliers, or failure of cooperation intentions. When the upstream enterprises of the logistics service supply chain cannot normally provide effective services to the downstream enterprises, the latter will not be able to purchase the required services, and the negative influence of the supply failure also will be transmitted along the logistics service supply chain.

If the logistics service integrators, as the core enterprise of a logistics service supply chain, cannot effectively integrate the logistics capacity to meet the needs of customers, it will lead to the operational risk. This may due to factors such as imperfection of their own system and poor regulation, thus causing sudden failure for logistics services integrator during the process of operation such as planning, organizing, implementing, and controlling logistics service capacities. Given a logistics service integrator is the core enterprise of a logistics service supply chain (Figure 1), its failure will lead to paralysis of the whole system of logistics service supply chain, which impedes business collaborations between the upstream and downstream demanders and suppliers of the logistics services. 




FIGURE 1: Structure of LSSC and risk scenarios.

Logistics capacity demand refers to the decision-making process of determining logistics demand by reverse transmission from the service demand of the downstream market to the upstream supply. Demand urgency is the demand risk caused by reverse transfer effect due to large fluctuation of logistics users' demand caused by factors such as market environment fluctuations, increased competition, lean management of enterprises and increased complexity of outsourcing, which is similar to the situation where the "bullwhip effect" in the product supply chain affects the decision-making parameters of upstream enterprises. It mainly includes two types: sudden increase in demand and sudden decrease in demand. A sudden change of demand caused by unexpected events will break the balance between supply and demand of original services in the logistics service supply chain, thus affecting the pricing and profit of upstream supply enterprises.

\section{Model Hypotheses and Problem Description}

According to the types of LSSC urgencies, the diffusion effect model indicating their effects is established. The premises and basic problems are presented as below.

\subsection{Premises}

Premise 1: information of the cost structure and market demand of LSSC is complete, and each player is a rational economic entity aiming to maximize its own profits.

Premise 2: capacity of customer demand $C\left(p_{2}\right)$ is the function of sales price $p_{2}\left(p_{2}>0\right)$ of the logistics service integrator, i.e., $C\left(p_{2}\right)=a-b p_{2}+\varepsilon$, where $a$ and $b$ are constants $(a>0, b>0)$ the probability density function $\varepsilon$ is a random variable of $f(\varepsilon)$, the mathematical expectation is $E(\varepsilon)=\varepsilon$, and the probability distribution is $F(\varepsilon)$. Assuming $\varepsilon$ and $p_{2}$ are independent of each other, $\varepsilon$ reflects the fluctuation of logistics demand caused by factors other than price, and the change of demand caused by factors related to price is mainly reflected in the parameter $p_{2}$.
Premise 3: the profits of functional logistics service provider $(A)$ and logistics service integrator $(I)$ are $G_{1}$ and $G_{2}$, then the profit functions of functional logistics service provider and logistics service integrator are $G_{1}=\left(p_{1}-p_{0}\right) \times C$ and $G_{2}=\left(p_{2}-p_{1}\right) \times C\left(p_{2}\right)$, where $p_{0}\left(p_{0}>0\right)$ is the price of logistics service subcontractor, $p_{1}\left(p_{1}>0\right)$ is the price of functional logistics service provider, and $C(C>0)$ represents the purchasing ability of logistics service integrator.

Premise 4: regardless of the logistics service failure, the service capacity of the functional logistics service provider can be completely achieved, and it can also be supplied according to the demand capacity of the logistics service integrator, i.e., $C=C\left(p_{2}\right)$.

Premise 5: the supply function of logistic capacity of subcontractors is $S=a_{s}+b_{s} p_{0}$, and the demand function of logistic capacity of customers is $C=a_{d}-$ $b_{d} p_{2} \cdot a_{s}$ and $a_{d}$ are constants $\left(a_{s}>0, a_{d}>0\right)$, and $b_{s}$ and $b_{d}\left(b_{s}>0, b_{d}>0\right)$ are the elasticity coefficients.

3.2. Problem Description. Without loss of generality, assuming the research object is three-level logistics service supply chain, the main participants are logistics service subcontractors $(S)$, functional logistics service providers $(A)$, logistics service integrators $(I)$, and logistics customers $(C)$, as shown in Figure 2. Each arrow line corresponds to a different logistics service product $L_{i}$, and the arrow direction indicates the flow direction of the service. LSSC decision sequence: first, the functional logistics service provider $(A)$ determines the retailer price of the service product $p_{1}$ according to its own benefit maximization and the service price of the logistics service subcontractor $p_{0}$; second, logistics service integrators $(I)$ determine the total capacity $C$ of logistics service integrators to purchase from suppliers according to logistics customer $(C)$ demand function $C\left(p_{2}\right)$, procurement price $p_{1}$, and profit maximization conditions. Therefore, it can be seen that this decision is, in essence, a Stackelberg game process. In the process of logistics service provision, the interaction between suppliers and integrators is observed according to the Stackelberg game, in which the 


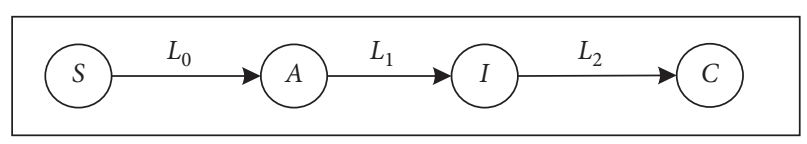

FIGURE 2: Game structure of the three-level logistics service supply chain.

functional logistics service provider $(A)$ is the leader and the logistics service integrator $(I)$ is the follower.

Firstly, logistics service integrator's decision-making process is considered first in the solution based on the Stackelberg game. Then the decision-making function of logistics service integrator $(I)$ is

$$
\max E G_{2}=E\left[\left(p_{2}-p_{1}\right) \times C\left(p_{2}\right)\right],
$$

where $E(\cdot)$ is the expectation of profit and is substituted into $C\left(p_{2}\right)=a-b p_{2}+\varepsilon$ :

$$
\begin{aligned}
\max E G_{2} & =E\left[\left(p_{2}-p_{1}\right) \times\left(a-b p_{2}\right)+\left(p_{2}-p_{1}\right) \times \varepsilon\right] \\
& =\left(p_{2}-p_{1}\right) \times\left(a-b p_{2}\right)+\left(p_{2}-p_{1}\right) \times E(\varepsilon) .
\end{aligned}
$$

Let $\mathrm{d} E G_{2} / \mathrm{d} p_{2}=0$ to obtain the price of logistics customers $p_{2}$, we have $p_{2}=\left(a+E(\varepsilon)+\mathrm{bp}_{1}\right) / 2 b$, while the ability of integrators to purchase from functional logistics service providers is $C=\left(a+E(\varepsilon)-b p_{1}\right) / 2$.

Secondly, decision-making of the functional logistics service provider $(A)$ is considered. In order to meet the procurement demand $C\left(p_{2}\right)$ of logistics service integrators, functional logistics service providers purchase logistics capacity $C$ from logistics function subcontractors $(S)$, and the provider's objective function is

$$
\left\{\begin{array}{l}
\max E G_{1}=E\left[\left(p_{1}-p_{0}\right) \times C\right]=\left(p_{1}-p_{0}\right) \times C, \\
C=\frac{a+E(\varepsilon)-b p_{1}}{2} .
\end{array}\right.
$$

Let $\mathrm{d} E G_{1} / \mathrm{d} p_{1}=0$, obtain the price of the logistics service integrator $p_{1}=\left(a+E(\varepsilon)+\mathrm{bp}_{0}\right) / 2 b$, the expected profits of functional logistics service providers and logistics service integrators are

$$
\left\{\begin{array}{l}
E G_{1}=\frac{\left(a+E(\varepsilon)-b p_{0}\right)^{2}}{8 b}, \\
E G_{2}=\frac{\left(a+E(\varepsilon)-b p_{0}\right)^{2}}{16 b} .
\end{array}\right.
$$

Thus, the final capacity price and service demand adopted by the logistics service integrator are

$$
\left\{\begin{array}{l}
p_{2}=\frac{3 a+3 E(\varepsilon)+b p_{0}}{4 b} \\
C\left(p_{2}\right)=\frac{a+E(\varepsilon)-b p_{0}}{4} .
\end{array}\right.
$$

\section{Risk Evolution Model of Logistics Service Supply Chain Involving the Urgencies}

This paper discusses the impact of the four kinds of urgencies, including price increase, capacity decrease, demand increase, and demand decrease, on the pricing, profit, and demand intensity of logistics service supply chain, as shown in Figure 3.

4.1. Increase of Supply Price. Under this circumstance, unexpected events lead to the continuous increase $\Delta p_{0}$ of logistics service supply price $p_{0}$, and the price and profit risk of downstream nodes caused by the sudden price change are focused. In accordance with the decision sequence, the elasticity coefficient of $p_{1}$ and $p_{2}$ to the supply price $p_{0}$ :

$$
\left\{\begin{array}{l}
\frac{\mathrm{d} p_{1} / p_{1}}{\mathrm{~d} p_{0} / p_{0}}=\frac{p_{0}}{(a+E(\varepsilon)) / b+p_{0}}, \\
\frac{\mathrm{d} p_{2} / p_{2}}{\mathrm{~d} p_{0} / p_{0}}=\frac{p_{0}}{3 \times(a+E(\varepsilon)) / b+p_{0}}, \\
p_{1}^{\prime}=\frac{a+E(\varepsilon)+b\left(p_{0}+\Delta p_{0}\right)}{2 b}, \\
p_{2}^{\prime}=\frac{3 a+3 E(\varepsilon)+b\left(p_{0}+\Delta p_{0}\right)}{4 b} .
\end{array}\right.
$$

Given $0<\left(\mathrm{d} p_{2} / p_{2}\right) /\left(\mathrm{d} p_{0} / p_{0}\right)<\left(\mathrm{d} p_{1} / p_{1}\right) /\left(\mathrm{d} p_{0} / p_{0}\right)<1$, if the price $p_{0}$ rises by $1 \%$, causing the increases of $p_{1}$ and $p_{2}$ less than $1 \%$, the diffusion intensity will be gradually weakened, and when the price rises $\Delta p_{0}$, the optimal price of the sales of functional logistics service providers to the logistics service integrators $p_{1}$ will increase $\Delta p_{0} / 2$, while the optimal price of the logistics service integrators $p_{2}$ will increase $\Delta p_{0} / 4$. It indicates that the price fluctuation caused by emergencies will be transmitted along the downstream direction of LSSC, and the buffer strength of each node is $50 \%$.

Similarly, the elasticity coefficients of the profit of the functional logistics service provider $G_{1}$ and of the logistics service integrator $G_{2}$ to price $p_{0}$ is

$$
\left\{\begin{array}{l}
\frac{\mathrm{d} G_{1} / G_{1}}{\mathrm{~d} p_{0} / p_{0}}=\frac{-2 p_{0}}{(a+E(\varepsilon)) / b-p_{0}} \\
\frac{\mathrm{d} G_{2} / G_{2}}{\mathrm{~d} p_{0} / p_{0}}=\frac{-2 p_{0}}{(a+E(\varepsilon)) / b-p_{0}}
\end{array}\right.
$$

from $C\left(p_{2}\right)>0$, we know that $\left(\mathrm{d} G_{1} / G_{1}\right) /\left(\mathrm{d} p_{0} / p_{0}\right)=$ $\left(\mathrm{d} G_{2} / G_{2}\right) /\left(\mathrm{d} p_{0} / p_{0}\right)<0$, suggesting that the fluctuation of supply price caused by emergencies reduces the downstream profits $G_{1}$ and $G_{2}$, and both have the same profit change rate. 




FIGURE 3: Risk evolution of logistics service supply chain involving the urgencies.

4.2. Decrease of Supply Quantity. Under this circumstance, Stackelberg's decision-making sequence is as follows: firstly, functional logistics service providers make decisions of $p_{1}$ based on the subcontractor supply function and profit maximization; secondly, logistics service integrators determine the optimal price $p_{2}$ according to logistics customers, providers $p_{1}$ and profit maximization. In this case, the expected profit function of logistics service integrator is

$$
\max G_{2}=\left(p_{2}-p_{1}\right) \times C\left(p_{2}\right) .
$$

Substitute $C=a_{d}-b_{d} p_{2}$ into equation (8), let $\mathrm{d} E G_{2} / \mathrm{d} p_{2}=0$ and obtain $p_{2}=\left(a_{d}+b_{d} p_{1}\right) / 2 b_{d}$. Provider profit function is

$$
\max G_{1}=\left(p_{1}-p_{0}\right) \times C .
$$

The results of solving parameters are as follows:

$$
\left\{\begin{array}{l}
p_{1}=\frac{\left(a_{d}-2 a_{s}\right) b_{d}+2 a_{d} b_{s}}{b_{d}\left(4 b_{s}+b_{d}\right)}, \\
p_{2}=\frac{1}{2 b_{d}}\left[a_{d}+\frac{\left(a_{d}-2 a_{s}\right) b_{d}+2 a_{d} b_{s}}{\left(4 b_{s}+b_{d}\right)}\right], \\
C=\frac{a_{d} b_{s}+a_{s} b_{d}}{4 b_{s}+b_{d}}, \\
p_{0}=\frac{a_{d}-4 a_{s}}{4 b_{s}+b_{d}}, \\
G_{1}=\frac{2\left(a_{d} b_{s}+a_{s} b_{d}\right)^{2}}{b_{d}\left(4 b_{s}+b_{d}\right)^{2}}, \\
G_{2}=\frac{G_{1}}{2}=\frac{\left(a_{d} b_{s}+a_{s} b_{d}\right)^{2}}{b_{d}\left(4 b_{s}+b_{d}\right)^{2}} .
\end{array}\right.
$$

For the analysis of the sudden decrease in the supply capacity of logistics service subcontractors caused by urgencies, hereby we study a typical case in which both $a_{s}$ and $b_{s}$ decreases. The change of supply curve is shown in Figure 4:

The sudden reduction of supply capacity can be decomposed into two basic processes: Process 1 refers to that the intercept of logistics subcontractor's supply capacity $S=$ $a_{s}+b_{s} p_{0}$ drops, $a_{s}$ turns to $a_{s}^{\prime}$, which makes the curve $S$ become $S^{\prime}$; Process 2 is a change in the price elasticity of $S=a_{s}+b_{s} p_{0}$ that causes the slope to go down and the supply



FIgURE 4: Sudden decrease in supply capacity.

to go from $S^{\prime}$ to $S^{\prime \prime}$. This research examines how these two scenarios influence downstream of the LSSC, thus influencing the demand and pricing of it.

Process 1: $a_{s}$ decreases $\Delta a_{s} b_{s}$ does not change. When supply becomes $S^{\prime}=a_{s}-\Delta a_{s}+b_{s} p_{0}$, the coefficient of demand elasticity is

$$
\frac{\mathrm{d} C / C}{\mathrm{~d} a_{s} / a_{s}}=\frac{a_{s} b_{d}}{a_{d} b_{s}+a_{s} b_{d}}<1 .
$$

Demand and supply change in the same direction, and the degree of change in demand is less than the degree of change in supply. The results of calculated parameters are as follows:

$$
\left\{\begin{array}{l}
\Delta C=\frac{\Delta a_{s} b_{d}}{4 b_{s}+b_{d}}, \\
\Delta p_{0}=\frac{4 \Delta a_{s}}{4 b_{s}+b_{d}}, \\
\Delta p_{1}=\frac{2 \Delta a_{s}}{4 b_{s}+b_{d}}, \\
\Delta p_{2}=\frac{\Delta a_{s}}{4 b_{s}+b_{d}}, \\
\Delta G_{1}=\frac{-2 \Delta a_{s} b_{d}\left(2 a_{d} b_{s}+2 a_{s} b_{d}-\Delta a_{s} b_{d}\right)}{b_{d}\left(4 b_{s}+b_{d}\right)^{2}} \\
\Delta G_{2}=\frac{-\Delta a_{s} b_{d}\left(2 a_{d} b_{s}+2 a_{s} b_{d}-\Delta a_{s} b_{d}\right)}{b_{d}\left(4 b_{s}+b_{d}\right)^{2}}
\end{array}\right.
$$

where $\Delta C<0, \Delta C<\Delta a_{s}$, that is, in Process 1, the continuous decrease of supply causes the decrease of demand, $\Delta p_{0}>\Delta p_{1}>\Delta p_{2}>0$ indicates that the continuous decrease of supply causes the price increase of the downstream in this case, and the lower increase rate of price along the LSSC. The supply chain node plays a buffer role in the price risk transmission.

Process 2: $b_{s}$ decreases $\Delta b_{s}, a_{s}$ does not change. That is, the supply capacity is $S=a_{s}+\left(b_{s}-\Delta b_{s}\right) p_{0}$, and we obtain 


$$
\Delta C=\frac{\Delta b_{s} b_{d}\left(4 a_{s}-a_{d}\right)}{\left(4 b_{s}-4 \Delta b_{s}+b_{d}\right)\left(4 b_{s}+b_{d}\right)} .
$$

From $\Delta b_{s}>0$ and $b_{s}>\Delta b_{s}$, we have $\left(4 b_{s}-4 \Delta b_{s}+\right.$ $\left.b_{d}\right)\left(4 b_{s}+b_{d}\right)>0$. The value of $\Delta C$ is determined by $\left(4 a_{s}-a_{d}\right)$, that is when $4 a_{s}>a_{d}, \Delta C>0$, whereas when $4 a_{s}<a_{d}, \Delta C<0$. The results of parameters are:

$$
\left\{\begin{array}{l}
\Delta p_{0}=\frac{4 \Delta b_{s}\left(a_{d}-4 a_{s}\right)}{\left(4 b_{s}-4 \Delta b_{s}+b_{d}\right)\left(4 b_{s}+b_{d}\right)}, \\
\Delta p_{1}=\frac{2 \Delta b_{s}\left(a_{d}-4 a_{s}\right)}{\left(4 b_{s}-4 \Delta b_{s}+b_{d}\right)\left(4 b_{s}+b_{d}\right)}, \\
\Delta p_{2}=\frac{\Delta b_{s}\left(a_{d}-4 a_{s}\right)}{\left(4 b_{s}-4 \Delta b_{s}+b_{d}\right)\left(4 b_{s}+b_{d}\right)}, \\
\Delta G_{1}=\frac{2 \Delta b_{s}\left(a_{d}-4 a_{s}\right)\left[a_{d} b_{d}\left(\Delta b_{s}-2 b_{s}\right)+4 a_{s} b_{d}\left(\Delta b_{s}-2 b_{s}\right)+8 a_{d} b_{s}\left(\Delta b_{s}-b_{s}\right)-2 b_{d}^{2} a_{s}\right]}{\left(4 b_{s}-4 \Delta b_{s}+b_{d}\right)^{2}\left(4 b_{s}+b_{d}\right)^{2}} \\
\Delta G_{2}=\frac{\Delta b_{s}\left(a_{d}-4 a_{s}\right)\left[a_{d} b_{d}\left(\Delta b_{s}-2 b_{s}\right)+4 a_{s} b_{d}\left(\Delta b_{s}-2 b_{s}\right)+8 a_{d} b_{s}\left(\Delta b_{s}-b_{s}\right)-2 b_{d}^{2} a_{s}\right]}{\left(4 b_{s}-4 \Delta b_{s}+b_{d}\right)^{2}\left(4 b_{s}+b_{d}\right)^{2}}
\end{array}\right.
$$

4.3. Changes in Logistics Demand. Similar to the change of supply capacity, it is necessary to analyze the scenario that both parameters $a_{d}$ and $b_{d}$ change in the demand function when LSSC logistics demand changes due to urgencies. Since the increase and decrease of demand are completely reverse processes in the function, this research only needs to study the continuous increase of demand. The change in demand increase is shown in Figure 5.

It can be divided into two basic processes: Process 1 is the change from $a_{d}$ to $a_{d}^{\prime}$ in the logistics customer demand
$C=a_{d}-b_{d} p_{2}$, which leads to the change of curve from $C$ to $C^{\prime}$; Process 2 is the change in elasticity of $C=a_{d}-b_{d} p_{2}$ that causes a change in the slope, which moves from $C^{\prime}$ to $C^{\prime \prime}$. This research studies how these two risks spread to the upstream of LSSC, and then affect the supply and pricing.

Process 1: $a_{d}$ changes $\Delta a_{d}, b_{d}$ does not change. That is, when the demand function is $C=a_{d}+\Delta a_{d}-b_{d} p_{2}$, we have

$$
\left\{\begin{array}{l}
\Delta C=\frac{\Delta a_{d} b_{s}}{4 b_{s}+b_{d}}, \\
\Delta p_{0}=\frac{\Delta a_{d}}{4 b_{s}+b_{d}}, \\
\Delta p_{1}=\frac{\Delta a_{d}\left(b_{d}+2 b_{s}\right)}{b_{d}\left(4 b_{s}+b_{d}\right)}, \\
\Delta p_{2}=\frac{\Delta a_{d} \times\left(3 b_{s}+b_{d}\right)}{b_{d}\left(4 b_{s}+b_{d}\right)}, \\
\Delta G_{1}=\frac{2 \Delta a_{d} b_{s}\left(2 a_{d} b_{s}+a_{s} b_{d}+\Delta a_{d} b_{s}\right)}{b_{d}\left(4 b_{s}+b_{d}\right)^{2}}, \\
\Delta G_{2}=\frac{\Delta a_{d} b_{s}\left(2 a_{d} b_{s}+a_{s} b_{d}+\Delta a_{d} b_{s}\right)}{b_{d}\left(4 b_{s}+b_{d}\right)^{2}},
\end{array}\right.
$$






Figure 5: Continuous increase in demand capacity.

where $\Delta C>0$ and $\Delta C<\left(\Delta a_{d} / 4\right)$. In Process 1 , the increase in demand causes the increase in upstream orders, but the increase is less than the change in demand. $\Delta p_{2}>\Delta p_{1}>\Delta p_{0}>0$ shows that the continuous increase of demand in this case causes the upstream price of LSSC to rise, and the further away from the demand increase point, the smaller the price changes. The supply chain node also plays a buffer role.

Process 2: $b_{d}$ decreases by $\Delta b_{d}, a_{d}$ does not change. When the demand function is $C=a_{d}-\left(b_{d}-\Delta b_{d}\right) p_{2}$, we obtain

$$
\Delta C=\frac{\Delta b_{d} b_{s}\left(a_{d}-4 a_{s}\right)}{\left(4 b_{s}+b_{d}\right)\left(4 b_{s}+b_{d}-\Delta b_{d}\right)} .
$$

From $\left|b_{d}\right|>\left|\Delta b_{d}\right|$, we know $4 b_{s}+b_{d}-\Delta b_{d}>0$, the sign of $\Delta C$ therefore depends on $\left(a_{d}-4 a_{s}\right)$, that is, when $a_{d}>4 a_{s}, \Delta C>0$; otherwise, $\Delta C<0$. The results of parameters are

$$
\left\{\begin{array}{l}
\Delta p_{0}=\frac{\Delta b_{d}\left(a_{d}-4 a_{s}\right)}{\left(4 b_{s}+b_{d}\right)\left(4 b_{s}+b_{d}-\Delta b_{d}\right)}, \\
\Delta p_{1}=\frac{\Delta p_{0}}{2}+\frac{\Delta b_{d} a_{d}}{2 b_{d}\left(b_{d}-\Delta b_{d}\right)}, \\
\Delta p_{2}=\frac{\Delta p_{0}}{4}-\frac{\Delta b_{d} a_{d}}{4 b_{d}\left(b_{d}-\Delta b_{d}\right)}, \\
\Delta G_{1}=\frac{2 \Delta b_{d} b_{s}\left(a_{d}-4 a_{s}\right)\left[\left(4 b_{s}+b_{d}\right)\left(a_{d} b_{s}+a_{s}\left(b_{d}-\Delta b_{d}\right)+\left(4 b_{s}+b_{d}-\Delta b_{d}\right)\left(a_{d} b_{s}+a_{s} b_{d}\right)\right]\right.}{\left(b_{d}-\Delta b_{d}\right)\left(4 b_{s}+b_{d}\right)^{2}\left(4 b_{s}+b_{d}-\Delta b_{d}\right)^{2}}+\frac{2 \Delta b_{d} b_{d}\left(a_{d} b_{s}+a_{s} b_{d}\right)^{2}}{\left(b_{d}-\Delta b_{d}\right)\left(4 b_{s}+b_{d}\right)^{2}} \\
\Delta G_{2}=\frac{\Delta b_{d} b_{s}\left(a_{d}-4 a_{s}\right)\left[\left(4 b_{s}+b_{d}\right)\left(a_{d} b_{s}+a_{s}\left(b_{d}-\Delta b_{d}\right)+\left(4 b_{s}+b_{d}-\Delta b_{d}\right)\left(a_{d} b_{s}+a_{s} b_{d}\right)\right]\right.}{\left(b_{d}-\Delta b_{d}\right)\left(4 b_{s}+b_{d}\right)^{2}\left(4 b_{s}+b_{d}-\Delta b_{d}\right)^{2}}+\frac{\Delta b_{d} b_{d}\left(a_{d} b_{s}+a_{s} b_{d}\right)^{2}}{\left(b_{d}-\Delta b_{d}\right)\left(4 b_{s}+b_{d}\right)^{2}} .
\end{array}\right.
$$

\section{Numerical Analysis}

In order to investigate the specific changes of Process 2 in Section 4.2 and Process 2 in Section 4.3, the following numerical example is used for further analysis. When verifying the model to analyze the evolutionary effect of emergency risk, and in order to avoid influence of special cases on the reliability of the model, this paper sets the basic parameters randomly within the model parameter constraints in Section 3.1, assuming $a_{s}=5, b_{s}=5, a_{d}=10$ or 25, $b_{d}=6, \Delta b_{s} \in[0,4]$. When $4 a_{s}>a_{d}, a_{d}=10$, when $4 a_{s}<a_{d}$, $a_{d}=25$. We first investigated Process 2 in Section 4.2, and the parameter changes are shown in Figures 6-9.

Comparing the trend of price change of each node in the supply chain of the logistics service with the price elasticity and profit of the logistics subcontractor when $4 a_{s}>a_{d}$ and $4 a_{s}<a_{d}$, it is found that the price and profit change of each node in the logistics service supply chain are related to the basic supply capacity of logistics subcontractors $a_{s}$ and the regular demand of logistics customers $a_{d}$. From Figure 6, we know that when $4 a_{s}>a_{d}$, the value of price change of each node is smaller than 0 , and with the increase of the value change of elasticity, the reduction rate of each node becomes more drastic. In this case, the values of profit change for both functional logistics suppliers $G_{1}$ and logistics service integrators $G_{2}$ are greater than 0 (Figure 7 ), and the greater the degree of supply price change is, the greater the increase rates of $G_{1}$ and $G_{2}$ are. It might be that the decrease of supply prices $p_{0}, p_{1}$, and $p_{2}$ leads to the increase of market customer demand, and compared with demand $a_{d}$, the basic capacity of the subcontractor of logistics service $a_{s}$ is relatively sufficient, so the profits of the functional logistics supplier $G_{1}$ and of the logistics service integrator $G_{2}$ in LSSC shows an upward trend. However, From Figure 8, we know that when $4 a_{s}<a_{d}$, due to the lack of basic capacity of logistics service subcontractors $a_{s}$, the price of each node shows an upward trend with the increase of the decreasing range of elasticity. At this time, the increase of logistics procurement price causes the negative profit change of the functional logistics suppliers $G_{1}$ and of the logistics service integrators $G_{2}$ 


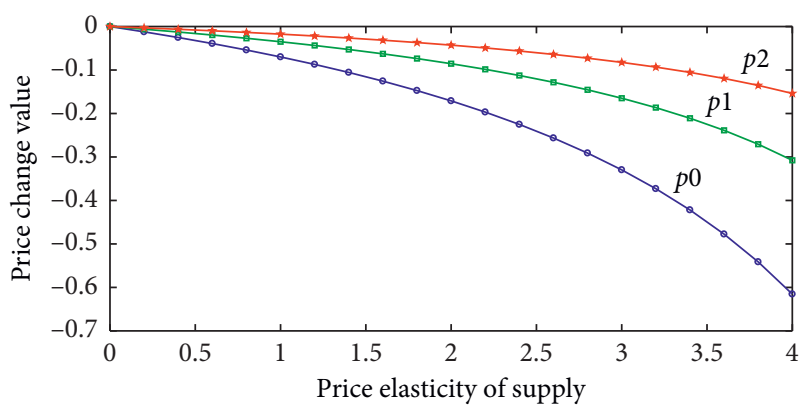

FIgURE 6: Changes in price parameters when $4 a_{s}>a_{d}$.

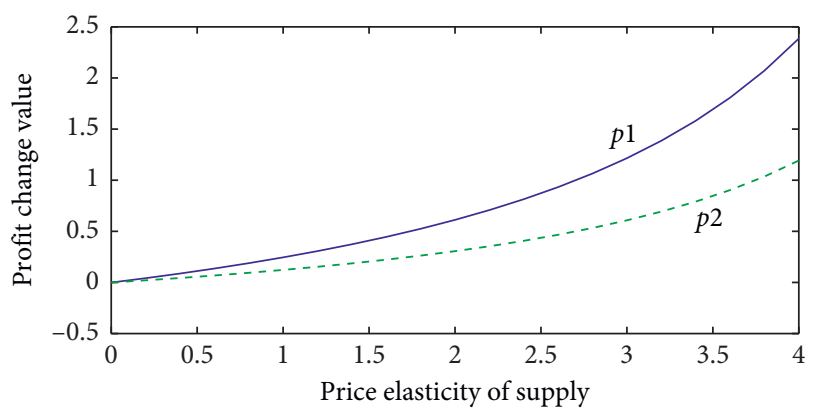

FIGURE 7: Changes in profit parameters when $4 a_{s}>a_{d}$.

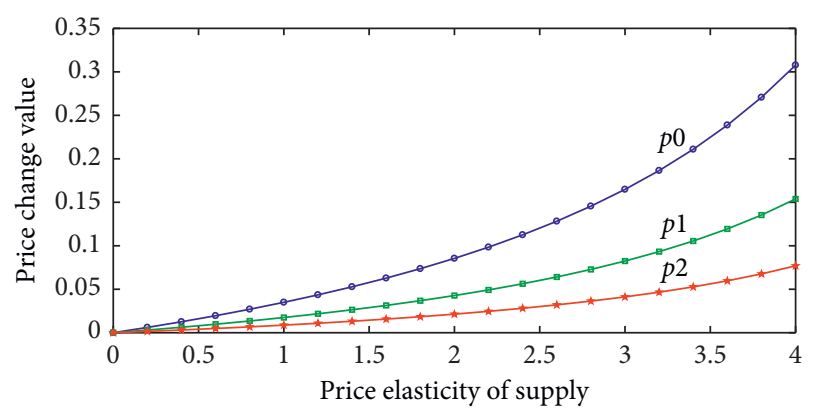

FIGURE 8: Changes in price parameters when $4 a_{s}<a_{d}$.

(Figure 9). Meanwhile, according to equation (14), the fluctuation of supply price elasticity will be transmitted along the LSSC, and the nodes' buffer strength of price is $50 \%$. It can be seen that for members of the logistics service supply chain, a stable purchasing price environment is a necessary condition for achieving ideal cooperation performance.

Similarly, the demand change Process 2 is further investigated, the basic parameters are set randomly; assuming $a_{s}=5, b_{s}=5, a_{d}=10$ or $25, b_{d}=6, \Delta b_{d} \in[0,4]$. When $4 a_{s}>a_{d}, a_{d}=10$, when $4 a_{s}<a_{d}, a_{d}=25$, the parameter changes are shown in Figures 10-13. From Figures 10 and 12 , we know that when the demand elasticity of logistics customers caused by urgencies increase continuously within a short time frame. In this case, in order to attract more customers, logistics service integrators adopt the sales strategy of price reduction, so when $4 a_{s}>a_{d}$ and $4 a_{s}<a_{d}$, the price changes of logistics services $p_{2}$ are negative and changes of the functional logistics service providers $p_{1}$ are

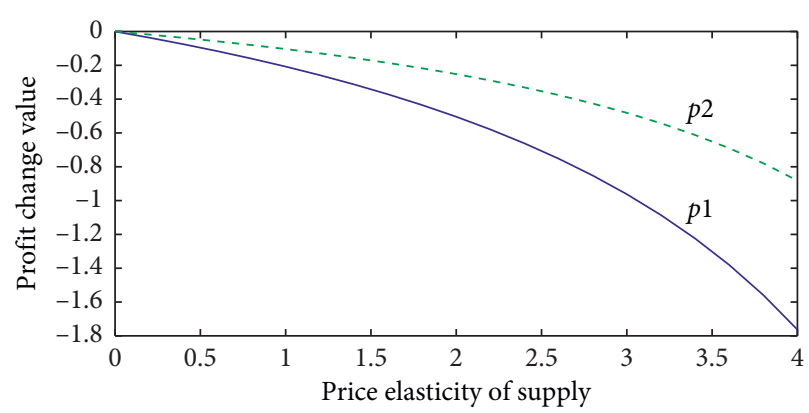

Figure 9: Changes in profit parameters when $4 a_{s}<a_{d}$.

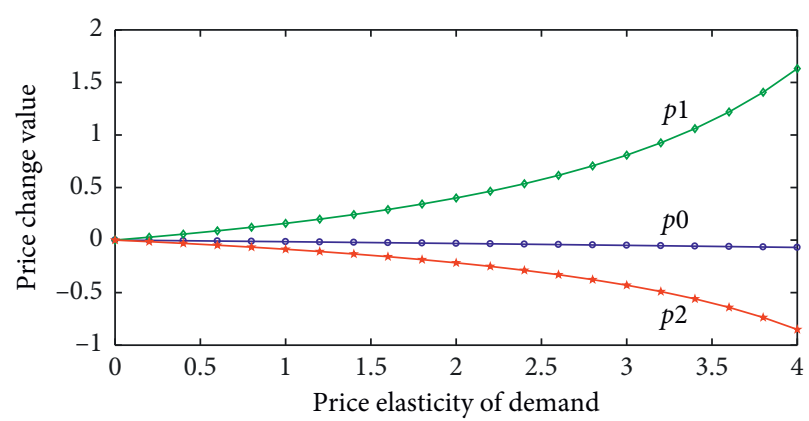

FIgURE 10: Change in price parameters when $4 a_{s}>a_{d}$.

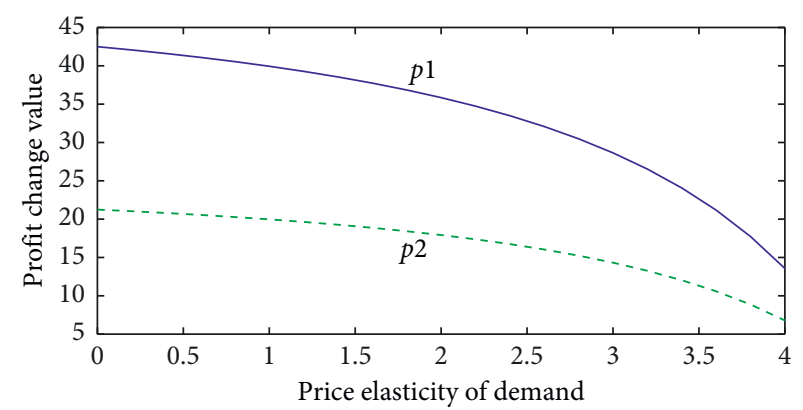

Figure 11: Changes in profit parameters when $4 a_{s}>a_{d}$.

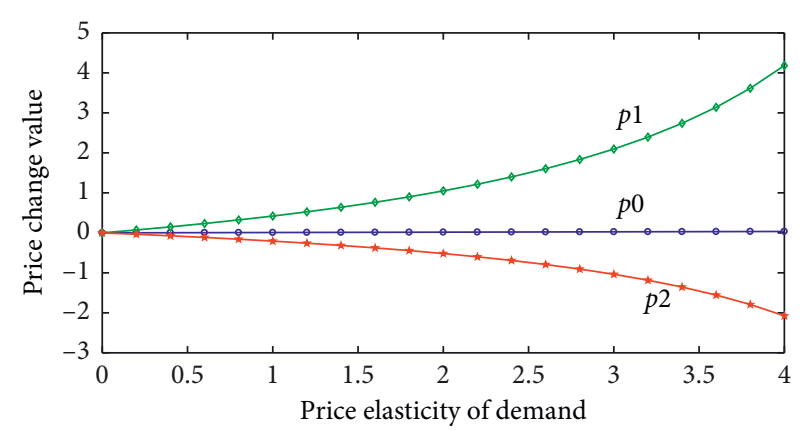

FIgURE 12: Changes in price parameters when $4 a_{s}<a_{d}$.

positive. Although the price of logistics service falls and the purchase price rises, the profit of logistics service integrators $G_{2}$ increases due to the increase of market demand and orders. At the same time, From Figures 11 and 13, we know that considering the increase of sales price $p_{1}$ and demand 


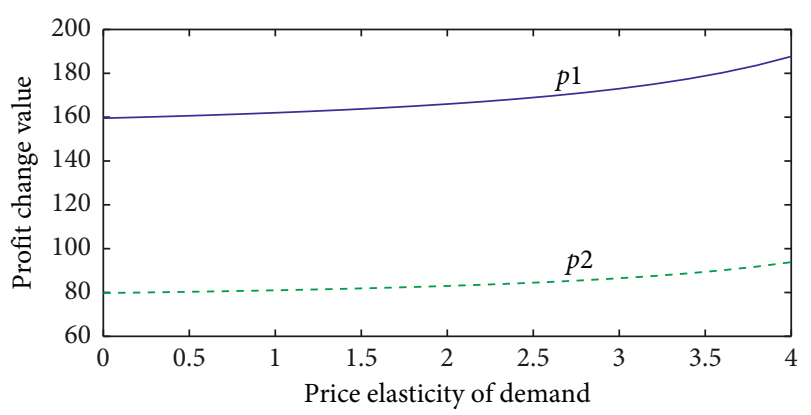

FIGURE 13: Changes in profit parameters when $4 a_{s}<a_{d}$.

intensity of functional logistics providers, the increase scale of profit $G_{1}$ is larger than that of profit $G_{2}$. From the perspective of supply chain operation, demand for sufficient capacity is the driving force of the entire logistics service supply chain. Uncertainty of logistics demand caused by urgencies will directly affect logistics service members' decision at all levels. The logistics service supply chain can formulate effective risk response strategies for demand urgencies by vertical coordination of the supply chain.

\section{Conclusion}

Based on Stackelberg game, the risk diffusion effect of different types of urgencies in the logistics supply chain has been studied. First, by constructing the urgency scenario framework that fits the structure of logistics service supply chain, the scenarios have been categorized into supply, operation, and demand urgency. Second, based on Stackelberg game model, the decision-making parameters of logistics service subcontractor $(S)$, functional logistics service provider $(A)$, logistics service integrator $(I)$, and logistics customer $(C)$ in a risk-free three-level logistics service supply chain have been solved. Third, a Stackelberg game model has been established for logistics service supply chain with urgencies and the impacts of different urgencies on pricing, profit, and demand intensity of the logistics service supply chain have been discussed. Fourth, based on numerical analysis, specific changes of parameters have been analyzed. The results indicate that the impacts and intensities of different logistics supply chain emergencies may differ. The fluctuation of supply price will cause the profit of the functional logistics service provider $G_{1}$ and the logistics service integrator $G_{2}$ to decrease with the same intensity. The decrease of supply will lead to the increase of downstream price of logistics service supply chain, and $\Delta p_{0}>\Delta p_{1}>$ $\Delta p_{2}>0$, which indicates that the nodes function as buffers of price risk transmission. The sudden increase (decrease) of demand will lead to the increase (decrease) of upstream order quantities, but the increase (decrease) intensity is less than the change intensity of customer demand. To sum up, this study has analyzed the urgent situations in the evolution of logistics service supply chain and the mechanism of main actors on a micro level. It finds that different types of urgencies have different effects on each node of the logistics service supply chain. Therefore, the main "players" should formulate different preventive measures according to their own business environment to reduce the impact and loss of risk events, and effectively improve the operation security and efficiency. First, when faced with the risk of supply urgencies in the logistics service supply chain affecting its supply capacity or supply channels, the multisource capability procurement model of the logistics service integrators can effectively reduce the decline of supply capacity and reduce the dependence of single supplier. Standing-by suppliers as reserved supply resource of integrators are only deployed when the main suppliers are at risk. It is of great significance to introduce standing-by suppliers to deal with the risk of supply capacity urgencies in the logistics service supply chain. Secondly, facing the price risk, the logistics service supply chain can first use the option contract procurement model that pays option premium to suppliers in advance during the sales period, and obtain the right to purchase the agreed quantity of products at the end of the period with the agreed price. The logistics service supply chain can also immediately purchase the required logistics service products in spot market. Finally, from the perspective of supply chain operation, demand for sufficient capacity is the driving force of the entire logistics service supply chain. Uncertainty of logistics demand caused by urgencies will directly affect logistics service members' decision at all levels. The logistics service supply chain can formulate effective risk response strategies for demand urgencies by vertical coordination of the supply chain. When there is a sudden increase of demand risk, the logistics service supply chain must build a logistics service time-effect model according to the priorities of customer to achieve optimal allocation of sudden demand capacity. While when there is a sudden decrease in demand, in order to maintain overall stability of the system, demand risk subsidies can be used to provide temporary income support to the members, which can effectively stabilize the original cost-profit balance of the logistics service supply chain system.

This study focuses on the "risk diffusion mechanism of logistics service supply chain in response to urgencies" and has obtained expected research results, but there still are some issues that need to be further studied and addressed. First, research on the elastic mechanism of logistics service supply chain considering the risk of urgencies is needed. Elasticity refers to the ability of the impacted operation system to quickly bounce back to the normal operation state [22]. The idea is to objectively involve the vulnerability of the supply chain and then embed the resilience into the supply chain design and balance the operation cost and risk event loss, so as to achieve the purpose of constant response to changes [23]. The supply chain with inlaid elastic mechanism demands better preparation, more flexible response, and faster recovery ability than competitors. Therefore, if the existence of urgency risk can be involved in the design and operation mechanism of logistics service supply chain, it will have critical theoretical value and practical significance for the capability to minimize the delivery failure of the service capacity after the occurrence of failure event and quickly restore to the normal operation state to improve customer satisfaction. Second, the risk simulation model of logistics service supply chain based on system dynamics can be 
introduced. The existing research on the risk system of logistics service supply chain is usually static and qualitative, and it is difficult to fully understand the complexity and dynamics of the strategy as a whole [1]. System dynamics studies the interdependence between the internal mechanism and behavior of the system through mathematical models and gradually discovers the causality based on morphological changes in the process of establishment and operation, which is suitable for the study of system coordination [24]. Vensim software can be applied to simulate and analyze basic data of the mechanism of logistics service supply chain risk, where the determination of factors such as individual members, cooperation structure, the proportion of the whole system, and cost control can be studied. The dynamic characteristics of a risk situation and its impact on the change of performance goals of the logistics service supply chain can be further discussed. Meanwhile, system parameters can be adjusted in order to optimize the LSSC operation performance. Structure of the LSSC under different risk circumstances can be analyzed to adjust the sensitivity of the optimal scheme and to provide feasible suggestions to cope with the challenges.

\section{Data Availability}

The data used to support the findings of this study are available from the corresponding author upon request.

\section{Conflicts of Interest}

The authors declare that they have no conflicts of interest.

\section{Acknowledgments}

The authors gratefully acknowledge the financial support provided by the Post-Doctoral Science Foundation of China under grant no. 2019M662109 and the Natural Science Foundation of China under grant no. 11871434.

\section{References}

[1] W. Liu, X. X. Liu, and X. Li, "The two-stage batch ordering strategy of logistics service capacity with demand update," Transportation Research Part E: Logistics and Transportation Review, vol. 83, pp. 65-89, 2015.

[2] H. Baharmand, T. Comes, and M. Lauras, "Managing incountry transportation risks in humanitarian supply chains by logistics service providers: insights from the 2015 Nepal earthquake," International Journal of Disaster Risk Reduction, vol. 24, pp. 291-307, 2017.

[3] R. Gedik, H. Medal, C. Rainwater, E. A. Pohl, and S. J. Mason, "Vulnerability assessment and re-routing of freight trains under disruptions: a coal supply chain network application," Transportation Research Part E: Logistics and Transportation Review, vol. 71, no. 3, pp. 45-57, 2014.

[4] A. Paloviita, T. Kortetmäki, A. Puupponen, and T. Silvasti, "Vulnerability matrix of the food system: operationalizing vulnerability and addressing food security," Journal of Cleaner Production, vol. 135, pp. 1242-1255, 2016.

[5] M. Christopher, Logistics \& Supply Chain Management, Pearson Higher Education, London, UK, 2016.
[6] D. Ivanov, Structural Dynamics and Resilience in Supply Chain Risk management, Springer International Publishing, Berlin, Germany, 2018.

[7] A. Qazi, A. Dickson, J. Quigley, and B. Gaudenzi, "Supply chain risk network management: a Bayesian belief network and expected utility based approach for managing supply chain risks," International Journal of Production Economics, vol. 196, pp. 24-42, 2018.

[8] R. Ojha, A. Ghadge, M. K. Tiwari, and U. S. Bititci, "Bayesian network modelling for supply chain risk propagation," International Journal of Production Research, vol. 56, no. 17, pp. 5795-5819, 2018.

[9] H. Liang'an, H. Guo, Y. Cheng, and X. Xie, “A new model for supply chain risk propagation considering herd mentality and risk preference under warning information on multiplex networks," Physica A: Statistical Mechanics and Its Applications, vol. 40, no. 4, pp. 20-31, 2019.

[10] P. D. Berger, A. Gerstenfeld, and A. Z. Zeng, "How many suppliers are best? A decision-analysis approach," Omega, vol. 32, no. 1, pp. 9-15, 2004.

[11] M. Kumar, P. Basu, and B. Avittathur, "Pricing and sourcing strategies for competing retailers in supply chains under disruption risk," European Journal of Operational Research, vol. 265, no. 2, pp. 533-543, 2018.

[12] K. Bimpikis, O. Candogan, and S. Ehsani, "Supply disruptions and optimal network structures," Management Science, vol. 31, no. 6, pp. 21-35, 2019.

[13] C. Wang and Z. Yin, "Using backup supply with responsive pricing to mitigate disruption risk for a risk-averse firm," International Journal of Production Research, vol. 56, no. 17, pp. 5660-5676, 2018.

[14] M. Fattahi, K. Govindan, and E. Keyvanshokooh, "Responsive and resilient supply chain network design under operational and disruption risks with delivery lead-time sensitive customers," Transportation Research Part E: Logistics and Transportation Review, vol. 101, pp. 176-200, 2017.

[15] D.-W. Kwak, Y.-J. Seo, and R. Mason, "Investigating the relationship between supply chain innovation, risk management capabilities and competitive advantage in global supply chains," International Journal of Operations \& Production Management, vol. 38, no. 1, pp. 2-21, 2018.

[16] D. Ivanov, A. Dolgui, and B. Sokolov, "The impact of digital technology and Industry 4.0 on the ripple effect and supply chain risk analytics," International Journal of Production Research, vol. 57, no. 3, pp. 829-846, 2019.

[17] X. Qi, J. F. Bard, and G. Yu, "Supply chain coordination with demand disruptions," Omega, vol. 32, no. 4, pp. 301-312, 2004.

[18] Y. Huang and Z. Wang, "Demand disruptions, pricing and production decisions in a closed-loop supply chain with technology licensing," Journal of Cleaner Production, vol. 191, pp. 248-260, 2018.

[19] B. Yan, Z. Jin, Y. Liu, and J. Yang, "Decision on risk-averse dual-channel supply chain under demand disruption," Communications in Nonlinear Science and Numerical Simulation, vol. 55, pp. 206-224, 2018.

[20] K. Rahmani and M. Yavari, "Pricing policies for a dualchannel green supply chain under demand disruptions," Computers \& Industrial Engineering, vol. 127, pp. 493-510, 2019.

[21] U. Jüttner, "Supply chain risk management," The International Journal of Logistics Management, vol. 16, pp. 120-141, 2005.

[22] B. Hull, "The role of elasticity in supply chain performance," International Journal of Production Economics, vol. 98, pp. 301-314, 2005. 
[23] K. Hjaila, Laínez-Aguirre, M. José, M. Zamarripa et al., "Optimal Integration of Third-Parties in a Coordinated Supply Chain Management Environment," Computers \& Chemical Engineering, vol. 86, pp. 48-61, 2016.

[24] J. P. Torres, Kunc, M. Kunc, and F. O"Brien, "Supporting strategy using system dynamics," European Journal of Operational Research, vol. 260, pp. 1081-1094, 2017. 